\title{
TRADITIONAL VERSUS EXPERT SYSTEMS: APPLICATIONS IN PROVIDING ACCOUNTING INFORMATION TO MANAGERS
}

Mr. Gary D. Buckner, Management Tool Company, Austin, Texas Vivek Shah, Southwest Texas State University

\begin{abstract}
Decisions involving the use of accounting data occur daily within most firms. Efforts to collect accounting data routinely focus on the creation of records describing what has happened, with whom, when it took place, and the value involved. The purpose of this article is to compare traditional systems and expert systems applications in providing accounting information to managers.
\end{abstract}

\section{Introduction}

Decisions which include accounting data as a basis are commonplace within most firms. Records describing what has happened, with whom, when it took place, and the value involved are created and maintained as the basic information source for the preparation of financial statements for external reporting purposes. From the same basic data, different reports can be developed to facilitate internal decision making by management. If the accounting data is presented to management in the same format as it is furnished to external groups, however, its usefulness to managers may decrease (11).

What unique factors characterize the internal information needs of an organization? Most managers require that data be assembled into a meaningful mosaic clarifying an organization's present position relative to its goals and the progress towards obtaining those goals (1). Historical documentation from a managerial accounting viewpoint is, therefore, useful only if it facilitates accomplishment of these tasks. Efforts to achieve the desired clarity can be summarized as follows (4):

* emphasize the future;

* de-emphasize generally accepted accounting principles;
* concentrate on relevance and flexibility of data;

* place less emphasis on precision and more emphasis on nonmonetary data;

* emphasize the segments of an organization, rather than just looking at the organization as a whole; and

* draw heavily from other disciplines (including economics, finance, statistics, operations research and organizational behavior) (4).

Computers are often used to enhance efforts to satisfy management's decision-oriented internal information needs because the processing speed of computers enables managers to obtain information promptly. The relatively low cost of computers makes them ideal accounting tools to assist in effective planning, directing and controlling (14).

Too often, potential users of computer generated information avoid using the output because of a perceived "take-it-or-leave-it approach" which these machines offer the user (9). The ever-present demand for numerical information has also been regarded as a major source of stress in today's business organizations. It needs to be recognized that while internal management may demand continuing analysis of numerical 
information from the accounting profession; however, a set of numbers on a page is not very impressive and has little impact on an audience of non-accountants, a class to which most managerial users of accounting data belong (10).

The purpose of this article is to explore the use of expert systems as potential managerial accounting decision-making tools. In accomplishing this task, the following issues will be addressed: (1) the qualities of information systems; (2) characteristics of more traditional software and general features of expert systems; and (3) conclusions concerning the impact of expert systems in making accounting decision.

\section{Qualities of Information Systems}

An information system, whether accounting or other, computer based or not, is a system which collects, classifies, stores, and communicates information to decision makers (2). During the last ten years, there has been increasing interest in developing computerized information systems designed to support managerial decision-making (7). It is axiomatic that managers must feel comfortable using a system in order for the process of decision-making to be facilitated. Failure to design specific information systems to be "user friendly" has often led to technically excellent systems becoming practically worthless because many managers simply would not use them. For an information system to be truly effective, it must produce information that is not only useful to managers, but is also obtainable in a way with which managerial personnel feel comfortable (16).

Furthermore, an evaluation of any information system is highly subjective because of the idiosyncratic nature of the decision-making process and the differing personalities of decision makers. Since specific objectives of information systems are often unique, it is not appropriate to broadly assign common objectives to all of the$\mathrm{m}$. Yet, certain "qualities inherent in information" as reflected in FASB 1980, are necessary for all systems in any decision-making situation (2). These qualities, with explanatory comments, are:

* Absence of Bias. In accounting, bias means a tendency to be consistently too high or too low. The goal in processing accounting data is to eliminate any bias before the data enters the information systems. Avoidance of bias in the manner by which an information system processes the data is equally as important.

* Comparability. Comparability is the quality of information that enables users to identify similarities in, and differences between, two sets of economic phenomena. Accounting data needs to possess this quality. An information system may be used to facilitate actual comparisons.

* Completeness. Completeness is the inclusion in reported information of everything that is necessary for faithful representation of the relevant phenomena. Accounting data should ideally have the quality of completeness before being input into an information system, but often this is not possible. An information system, on the other hand, should provide only the information which it has been designed to provide. Thus, if an information system is to provide only summary data, then completeness would involve providing that summary data only.

* Conservatism. Conservatism is the prudent reaction to uncertainty in order to be sure that uncertainty and risks inherent in business situations are adequately considered. Results produced by an information system using accounting data should enable managers to do so.

* Consistency. Consistency is conformity from period to period and within a period with unchanging policies and procedures. Accounting data should have the quality of consistency; this does not mean, however, that users of accounting data should not have flexibility in determining the way in which their information systems utilize the inputed accounting data.

* Feedback Value. Feedback value is the quality of information that enables users to confirm or correct prior expectations. The results produced by information systems utilizing accounting data need to have feedback value so managerial personnel can optimally use the accounting data.

* Materiality. Materiality is the magnitude of an omission or misstatement of accounting information that, in the light of surrounding circumstances, makes it probable that the judgment of a reasonable person relying on the information would have been changed or influenced by the omission or misstatement. Accounting data that is inputed into a manager-oriented information system should be focused on the deci- 
sion-making process as it relates to materiality.

* Neutrality. Neutrality is the absence in reported information of bias intended to attain a predetermined result or to induce a particular mode of behavior. Both inputing and processing of accounting data need to be done in a manner which ensures neutrality.

* Predictive Value. Predictive value is the quality of information that increases the likelihood of users to correctly forecast the outcome of present or future events. The results produced by an information system utilizing accounting data need to have predictive value so managerial personnel can optimally use the accounting data.

* Relevance. Relevance is the capacity of information to make a difference in a decision by helping users to form predictions about the outcomes of past, present, and future events or correct prior expectations. Accounting data should have relevance. Information systems need to be designed to use the accounting data in a manner which produces results of relevance to managerial personnel.

* Reliability. Reliability is the characteristic of information that assures that information is reasonably free from error and bias and also faithfully represents what it purports to represent. Accounting data needs to be collected and input into the decision-making process in such a way that its reliability is maintained. Information systems need to be designed to maintain the reliability of processed accounting data and to provide reliable results to managers.

* Timeliness. Timeliness is the characteristic of having information available to a decision maker before it loses its capacity to influence decisions. Both timely input of accounting data and timely output of results from information systems are needed.

* Understandability. Understandability is the quality of information that enables users to perceive its significance. Both results produced by information systems and the raw accounting data must be obtainable by managers in a manner which they can understand.

* Validity. This quality is the correspondence or level of agreement between a measure of description and the phenomenon that it purports to represent. Accounting data needs to have validity before it's input into an information system. Results produced by the system must ensure that representational faithfulness is maintained.

* Verifiability. Verifiability is the ability through consensus among measures to ensure that information represents what it purports to represent. Results produced by information systems as well as the raw accounting data must be verifiable.

\section{Characteristics of Traditional Software}

Traditional software used by business organizations can provide support in the following four major areas (1):

* information creation, whereby raw data are assembled into a more informational form;

* intelligence dissemination, whereby the created information is used to generate organizational options;

* communication, whereby these options are rapidly transmitted to and from decision-makers throughout the organization; and

* control and documentation, through which adherence to the organization's goals can be tracked for appropriate feedback and predictable, accurate results.

However, there are certain limiting features common to use of most traditional software. These characteristics include (15):

* all the decision rules used in these softwares must be reduced to formulas and the input data must be numbers;

* there is only one solution for any set of data;

* the computer cannot be used to reach any conclusion if any of the input data is missing;

* the solution is given with no indication as to the degree of uncertainty about it and, even when a solution is stated in probabilistic terms, the user is more often than not led to think it is the one and only probability that fits the data;

* one generally accepted way to solve a problem is stated; and

* the tasks change relatively little over time.

These characteristics have led to a predominantly input-to-output format style of programming, irrespective of which particular programming language is used. This approach requires that the input data be kept strictly separated fro$\mathrm{m}$ the algorithms (or coded instructions) which comprise the computer program. The program- 
mer's task was to develop the algorithms needed to transform the input data into the pre-determined output format. These techniques have led to highly specific and thus limited application programs being developed. The resulting output from information systems possessing these characteristics is often provided to users in a "takeit-or-leave-it" manner (9).

\section{Expert Systems}

An expert system employs the set of decision rules used by professionals in a discipline. An inference rule is a logical rule such as "if this is true do this else do this," or "when this condition exists take this route," etc. An expert system performs logical analysis of a specialized, limited body of information to provide suggestions, or recommendations, often through interaction with a human user (manager), until a viable solution is found.

The goal in the development of an expert system is to create programs which go beyond the limits of traditional programming. In addition to having an expert system solve problems, it allows managers to learn and receive explanations about the results of the problem-solving process since expert systems are usually built to explain the lines of reasoning that led to their decisions. The explanatory capabilities of expert systems range from basic back-tracking through a problem to a multi-level explanation that describes how and why the system reached a particular conclusion. This feature helps an organization because it assists managers in making decisions and understanding the reason why certain paths (decisions) were selected while others were rejected.

Traditionally, numerical problems were the only type of problems to be solved. Symbolic processing as well as numeric processing are now the focus of expert systems. Symbolic processing involves using symbols which aid in the interpretation of solutions. The purpose of symbolic processing is to evoke some pre-determined behavior and to give relevance to proposed solutions. For an expert system to be useful in the accounting environment, it should process both symbolic and numerical data as well as make intelligent decisions without having all the necessary, requisite, or required facts.
The following is a likely exchange between a user and an expert system (8):

* the user poses a problem to the expert system;

* the expert system asks for more information in order to fully understand the problem;

* the user asks why the additional information is needed;

* if asked why additional information is needed, the expert system provides an explanation;

* to solve the problem, the expert system uses its "inference engine" to process predetermined "if-then" rules and retrieves a conclusion;

* the expert system provides a conclusion to the user;

* the user inquires as to how the conclusion was obtained;

* the expert system provides the line of reasoning leading to the conclusion;

* human evaluates logic and can accept or reject the solution.

Such human-machine (or actually humansoftware) interaction often allows people to avoid the annoying "take-it-or-leave-it" approach which users often associate with using computers today. Since users often perceive that it is possible to work with the expert system using the same problem-solving paradigm (one which simulates the thought processes of human experts), they often take a more active role in the computerbased problem-solving process.

However, there are certain important commonalties in the situations found to be appropriate for expert systems utilization. Highlights of these characteristics are (15):

* there are many possible solutions and it would take too long for humans to thoroughly examine each one;

* the problem-solving expertise that is needed is conceptual in nature and cannot be simply reduced to numbers;

* input data is often incomplete, uncertain, subjective, inconsistent, and/or subject to change;

* the conclusions reached will often be uncertain and it is acceptable for the expert system to rank conclusions by their likelihood of being correct;

* human experts may disagree on how to solve the problem and therefore rule-based procedural flexibility must be allowed; 
* the task is always changing and evolving, and thus rule-based flexibility must again be faciliated; or

* the cost of a late or poor decision is very high.

There are also barriers to the development of expert systems. Barriers of significance to managerial personnel would likely include (15):

* determining what knowledge needs to be built into the system in order to accomplish a desired task;

* reconciling experts who disagree or approach the same task in different ways;

* lack of knowledge acquisition tools that are flexible and easy enough to be used by nonprogramming managers;

* the shortage of experienced support staff to assist in the development and maintenance of expert systems.

It is therefore appropriate to ask, "under what conditions would the relationship between costs and benefits arising from the use of an expert system likely merit the use of such an information system?" In this regard, an expert system would be most practical when one or more of the following conditions exist (15):

* the required expertise is not always available, is very expensive, or is rare;

* expertise is needed to augment the knowledge of junior personnel;

* there are too many factors or possible solutions for humans to keep in mind at once, even when the problem is broken down into smaller pieces;

* decisions must be made under pressure and omission of even a single factor could be costly;

* a huge amount of data must be evaluated and analyzed before the decision-making process can even begin;

* one type of expertise must be made available to people in a different field so they can make better decisions;

* there is rapid turnover of personnel, a constant need to train new people, and training is expensive and time consuming; or

* the problem requires use of a knowledge base and cannot be handled by a conventional computational approach.

\section{Conclusions}

The following conclusions have been derived from the preceding considerations and factors:

* Managers can use computers to create information, disseminate intelligence, communicate with other organizational members, and for control and documentation purposes. Other productive uses of computer resources may also arise. If effectively used, these machines can become excellent managerial accounting decision tools.

* Expert systems may be useful as managerial accounting decision tools because: their interactive mode of operation may lead to managerial personnel becoming more receptive to the use of computers to facilitate decision-making since the output from such systems may be obtainable in a more understandable and comfortable manner; and the expertise designed into these systems may enhance the timeliness and conservative obtaining of feedback and predictive value from accounting data.

* Use of expert systems will still require that raw accounting data be collected and input into the system in an unbiased, neutral, timely, and consistent manner. While the completeness of data is still a desired quality, it may or may not be a vital factor when using an expert system. Processing of raw data and production of results by expert systems will need to be accomplished in a similar manner.

* Managers will still need to determine the materiality, validity, reliability, and relevance of output produced by expert systems. The ability of expert systems to explain how they reached particular conclusions is potentially the most significant feature of these systems in regards to these qualities of the information provided to management.

* Managers will likely wish to make comparisons between the specific cost-benefit relationships of traditional vis-a-vis expert systems for their firms before fully committing themselves to using either type of system as the preferred computer-based managerial accounting decision tool.

Continued on Page 79 